\title{
ALGEBRAIC GROUP AUTOMORPHISMS HAVING FINITE FIXED POINT SETS
}

\author{
DAVID J. WINTER ${ }^{1}$
}

1. Introduction. The main conclusions of this paper are given in Theorems 7, 10, 11 and 12. These theorems describe some of the structure of a connected linear algebraic group $G$ having a birational automorphism $\sigma$ such that the subgroup $F_{G}(\sigma)$ of fixed points of $\sigma$ in $G$ is finite. (Some of this structure is described in [3] in the case where $F_{G}(\sigma)=\{1\}$.)

Algebraic group automorphisms having only finitely many fixed points occur significantly more frequently than do those which keep fixed only the identity, and they occur under less restrictive conditions. (One simple indication of this is that over the field of complex numbers, there are tori of positive dimension which admit birational automorphisms of prime period having only finitely fixed points, but every such automorphism has nonidentity fixed points.)

The proof of Theorem 7 is heavily dependent upon Lemmas 2 and 6. Lemma 6 is also important for the proofs of Theorems 10, $11,12$.

2. Preliminaries. In this paper, $G$ is a connected linear algebraic group over an algebraically closed field of characteristic 0 or $p>0$ and $\sigma$ is a birational automorphism of $G$. If $G$ is a normal subgroup of a group $K$, then for $x$ in $K$, Int $_{G} x$ is the automorphism of $G$ defined by: $\operatorname{Int}_{G} x(g)=x^{-1} g x$ for $g$ in $G$.

Lemma 1. Let $G$ be semisimple and let $\sigma$ be a birational automorphism of $G$. Then there is a linear algebraic group $K$ containing $G$ as a closed normal subgroup of finite index and an element $s$ in $K$ such that $\sigma=\operatorname{Int}_{G} s$.

Proof. Aut $G / \operatorname{Int}_{G} G$ is finite $[2, \S 17-07]$. It follows that for some integer $n, \sigma^{n}$ lies in $\operatorname{Int}_{G} A$ where $A$ is a closed connected divisible abelian subgroup of $G$ having the property that $\sigma$ commutes with the elements of $\operatorname{Int}_{G} A$. (For if $\sigma^{k}=\operatorname{Int}_{G} s$ and if $B$ denotes the closed subgroup of $G$ generated by $s$, then $B$ is abelian and, as is easily seen, $\sigma$ commutes with each element of $\operatorname{Int}_{G} B$; moreover for some $j, \sigma^{k j}$

Received by the editors May 13, 1966.

1 Part of the work described here was done on a National Science Foundation Fellowship and is part of a dissertation directed by Professor George Seligman and presented for the degree of Doctor of Philosophy in Yale University. The remaining work was supported by research grant NSF-GP-4017. 
lies in Int ${ }_{G} B_{0}$, where $B_{0}$ is the connected component of the identity in $B$ and has the form $B_{0}=T U$ (direct), $T$ being a torus and $U$ a closed connected unipotent subgroup of $G$; therefore we can let $A=B_{0}$ if the characteristic is $0, A=T$ if the characteristic is $p>0$.) Consequently, there exists $\tau$ in Aut $G$ such that $\tau^{n}=1, \sigma \tau=\tau \sigma$, and $\sigma \in \tau \operatorname{Int}_{G} G$. (For if $\sigma^{n}=\operatorname{Int} x^{n}$ with $x$ in $A$, we can let $\tau=\sigma \operatorname{Int}_{G} x^{-1}$.) Let $K=\langle\tau\rangle G$ (semidirect). Then $K$ can be given the structure of a linear algebraic group containing $G$ as a closed subgroup, since $(K: G)$ is finite (e.g. by way of the representation of $K$ induced by $G)$. Choosing $y$ in $G$ such that $\sigma=\tau \operatorname{Int}_{G} y$, we have $\sigma=\operatorname{Int}_{G} s$ where $s=\tau y$ in $K$.

We need the following lemma (see also Theorem 2 of [6]):

Lemma 2. Let $G$ be semisimple. Let $\tau$ be a birational automorphism of $G$ which keeps stable a maximal torus $T$ of $G$ and a Borel subgroup $B$ of $G$ containing $T$. Then $\operatorname{dim} F_{G}(\tau) \geqq 1$ if $\operatorname{dim} G \geqq 1$.

Proof. Suppose that $\operatorname{dim} G \geqq 1$. The cyclic group $\langle\tau\rangle$ generated by $\tau$ acts in the group $T^{*}$ of rational characters of $T$ and keeps stable the subset $S$ of fundamental roots of $T$ with respect to $B$. Let $m$ be the index in $T^{*}$ of the subgroup generated by $S$. Let $t$ be an element of finite order such that $\alpha(t)=\beta(t)$ whenever $\alpha$ and $\beta$ are in the same orbit of $\langle\tau\rangle$ in $S$. Then $\alpha(\tau(t))=\alpha(t)$ for $\alpha$ in $S$. Thus $\chi^{m}(\tau(t))=\chi^{m}(t)$ for $\chi$ in $T^{*}$. Thus $\chi\left(\tau\left(t^{m}\right)\right)=\chi\left(t^{m}\right)$ for $\chi$ in $T^{*}$ and $\tau\left(t^{m}\right)=t^{m}$ since $T^{*}$ separates points. The order of $t$ (and hence of $t^{m}$ ) can be taken to be arbitrarily large (since $S$ is a linearly independent subset of $T^{*}$ ). Thus $\operatorname{dim} F_{T}(\tau) \geqq 1$.

LEMMA 3. Let $\mathbb{S}$ be a p-group having a normal subgroup $\mathfrak{S}$ which is infinite and has a faithful finite dimensional representation $\rho$ over a field of characteristic $p$. Then the centralizer in $\mathfrak{S}$ of each element $g$ of is infinite.

Proof. Let $g$ be an element of $\mathfrak{F}$. Let $\mathfrak{M}=\langle g\rangle \mathfrak{S}$. Then $(\mathfrak{T}$ : $\mathfrak{S})$ is finite and $\rho$ induces a faithful finite dimensional representation of $\mathfrak{T}$. Thus we may assume without loss of generality that $\mathfrak{T}$ is a linear group. This group consists of unipotent transformations since $\mathfrak{T}$ is a p-group. Thus $\mathfrak{T}$ is nilpotent by Kolchin's theorem [5, pp. 775 and 776]. Let $\mathfrak{S}_{0}$ be the (Zariski-) connected component of the identity in $\mathfrak{S}$. Then $\mathfrak{S}_{0}$ is a normal subgroup of $\mathfrak{T}$ of finite index, whence $\mathfrak{S}_{0}$ is infinite. For $S \subseteq \mathfrak{N}$, let $g_{0}(S)=S, g_{1}(S)=(g, S), \cdots$, $g_{i+1}(S)=\left(g, g_{i}(S)\right) \quad\left((g, h)=g^{-1} h^{-1} g h\right)$. Since $\mathfrak{T}$ is nilpotent we can find $i$ such that $g_{i+1}\left(\mathfrak{S}_{0}\right)=\{1\}$ and $g_{i}\left(\mathfrak{S}_{0}\right) \neq\{1\}$. Since $\mathfrak{S}_{0}$ is con- 
nected, $g_{i}\left(\mathfrak{S}_{0}\right)$ is connected. Thus $g_{i}\left(\mathfrak{S}_{0}\right)$ is an infinite subset of $\mathfrak{S}$ centralizing $g$. Thus the centralizer in $\mathfrak{S}$ of an arbitrary element $g$ of $(5)$ is infinite.

3. Solvability of $G$ when $F_{G}(\sigma)$ is finite. For $x, g$ in $G$, let $x_{\sigma} g$ $=\sigma\left(g^{-1}\right) x g$. $G$ acts on itself on the right as a group of birational transformations, an element $g$ in $G$ sending $x$ into $x_{\sigma} g$. Since $F_{G}(\sigma)$ $=\left\{g \mid 1_{\sigma} g=1\right\}$, an application of a dimension theorem [1, Theorem 2, p. 106] yields:

Lemma 4. $F_{G}(\sigma)$ is finite if and only if $1_{\sigma} G=\left\{1_{\sigma} g \mid g \in G\right\}$ is dense in $G$.

An immediate consequence of this is:

Lemma 5. Let $R$ be a closed connected characteristic subgroup of $G$. Define $\bar{G}=G / R$ and let $\bar{\sigma}$ denote the automorphism of $\bar{G}=G / R$ induced by $\sigma$. Then if $F_{G}(\sigma)$ is finite, $F_{\bar{G}}(\bar{\sigma})$ is finite.

If $\sigma$ has finite order, the semidirect product $\langle\sigma\rangle G$ of the cyclic group $\langle\sigma\rangle$ generated by $\sigma$ with $G$ may be regarded as a linear algebraic group containing $G$ as a closed normal subgroup of finite index (by way of the representation of $\langle\sigma\rangle G$ induced by $G$ ). Elements $\tau$ of $\langle\sigma\rangle G$ are regarded as automorphisms of $G$ as follows: $\tau(g)=\tau^{-1} g \tau$ for $\tau$ in $\langle\sigma\rangle G$ and $g$ in $G$.

Lemma 6. Let $\sigma$ have finite order $n$. Suppose that $F_{G}(\sigma)$ is finite. Define $K=\langle\sigma\rangle G$ (semidirect). Then for $\tau$ in the subset $\sigma G$ of $K$, the exponent of $F_{G}(\tau)$ divides $n$.

Proof. $1{ }_{\sigma} G=\left\{\sigma^{-1} g^{-1} \sigma g \mid g \in G\right\}$ is dense in $G$ by Lemma 4 . Thus $\sigma\left(1_{\sigma} G\right)=\left\{g^{-1} \sigma g \mid g \in G\right\}$ is dense in $\sigma G$. If $N=\left\{\tau \in \sigma G \mid \tau^{n}=1\right\}$, then $N$ is closed and $\left\{g^{-1} \sigma g \mid g \in G\right\} \subseteq N$. Thus $N=\sigma G$ and $\tau^{n}=1$ for $\tau$ in $\sigma G$. Thus for $\tau$ in $\sigma G$ and $g$ in $F_{G}(\tau), 1=(\tau g)^{n}=\tau^{n} g^{n}=g^{n}$. Thus the exponent of $F_{G}(\tau)$ divides $n$.

Theorem 7. Let $F_{G}(\sigma)$ be finite. Then $G$ is solvable.

Proof. Suppose first that $G$ is semisimple. Then by Lemma 1, there is a linear algebraic group $K$ containing $G$ as a closed normal subgroup of finite index and an element $s$ in $K$ such that $\sigma=\operatorname{Int}_{G} s$. If $(K: G)=m$, then $F_{G}(\sigma)$ contains $s^{m}$ and $s^{m}$ has finite order. Thus $\sigma$ has finite order. Thus we may take $K$ to be the semidirect product $\langle\sigma\rangle G$ described in Lemma 6 . Let $T$ be a maximal torus of $G, B$ a Borel subgroup of $G$ containing $T$. Since $\operatorname{Int}_{G} G$ acts transitively on the set of Borel subgroups of $G$, and since $\operatorname{Int}_{B} B$ acts transitively on the set of maximal tori of $B$, there exists $\tau$ in $\sigma G$ such that $B$ and $T$ are 
$\tau$-stable. If $\operatorname{dim} G \geqq 1$, then $\operatorname{dim} F_{T}(\tau) \geqq 1$ by Lemma 2 . But the exponent of $F_{G}(\tau)$ is finite by Lemma 6 . Thus $\operatorname{dim} G=0$ and $G=\{1\}$. We now consider the general case and let $R$ be the radical of $G$. Define $\bar{G}, \bar{\sigma}$ as in Lemma 5. Then $F_{\bar{G}}(\bar{\sigma})$ is finite since $F_{G}(\sigma)$ is finite (Lemma 5). Thus $\bar{G}=\{1\}$ by the preceding discussion. Thus $G$ is solvable.

\section{General structure of $(G, \sigma)$ when $F_{G}(\sigma)$ is finite.}

Proposition 8. Let $F_{G}(\sigma)$ be finite. Then $1_{\sigma} G=G$ and $\sigma$ keeps stable a maximal torus of $G$.

Proof. $G$ is solvable by Theorem 7. If $G$ is abelian, then the mapping sending $g$ into $1_{\sigma} g$ is a rational homomorphism from $G$ into $G$; and therefore $1_{\sigma} G$ is closed. But $1_{\sigma} G$ is dense in $G$ by Lemma 4 . Thus $1_{\sigma} G=G$ if $G$ is abelian.

The proof continues by induction on $\operatorname{dim} G$. Let $R$ be a closed connected characteristic abelian subgroup of $G$ of positive dimension (e.g. the next to last term of the derived series of $G$ ). Define $\bar{G}, \bar{\sigma}$ as in Lemma 5. Then $F_{\bar{G}}(\bar{\sigma})$ is finite by Lemma 5 and $\bar{G}=\overline{1}_{\bar{\sigma}} \bar{G}$ by induction. Thus for $g$ in $G$, there exists $x$ in $G$ such that $\bar{g}=\overline{1}_{\bar{\sigma}} \bar{x}$. Then $\overline{1}=\bar{g}_{\bar{\sigma}} \bar{x}^{-1}=\left[g_{\sigma} x^{-1}\right]-$ and $g_{\sigma} x^{-1}$ is in $R$. Since $R$ is abelian, $R=1_{\sigma} R$. Thus $g_{\sigma} x^{-1}=1_{\sigma} y$ for some $y$ in $R$. Then $g=\left(g_{\sigma} x^{-1}\right)_{\sigma} x=\left(1_{\sigma} y\right)_{\sigma} x=1_{\sigma}(y x)$ and $g$ is in $1_{\sigma} G$. Thus $G=1_{\sigma} G$.

Now a standard argument shows that $\sigma$ keeps stable a maximal torus of $G$. Let $T$ be a maximal torus of $G$. Then $\sigma(T)=x^{-1} T x$ for some $x$ in $G$. Since $G=1_{\sigma} G, x=g \sigma\left(g^{-1}\right)$ for some $g$ in $G$. Now $\sigma\left(g^{-1} T g\right)$ $=\sigma(g)^{-1} \sigma(T) \sigma(g)=\sigma(g)^{-1}\left(x^{-1} T x\right) \sigma(g)=g^{-1} T g$ (since $x \sigma(g)=g$ ), and $g^{-1} T g$ is $\sigma$-stable.

Leмma 9. Let $F_{G}(\sigma)$ be finite and let $\sigma$ have finite order. Let $\sigma_{s}$ be the p-regular part of $\sigma$. Then $F_{G}\left(\sigma_{s}\right)$ consists of semisimple elements.

Proof. $G$ is solvable by Theorem 7. Thus $G=T U$ where $T$ is a maximal torus of $G$ and $U$ is a closed connected characteristic unipotent subgroup of $G$. Let $\sigma=\tau \mu$ where $\tau, \mu$ are the $p$-regular and $p$-singular components of $\sigma$ respectively. Then $V=F_{U}(\tau)$ is connected (by $[2, \S 6-02$, Corollary 2], since $\sigma$ is of finite order and $\langle\sigma\rangle G$ can be given the structure of an algebraic linear group containing $G$ as a closed subgroup) and $\mu$-stable. If $\operatorname{dim} V \geqq 1, F_{V}(\mu)$ is infinite (apply Lemma 3 to $\$=\langle\mu\rangle V$ if the characteristic is $p>0$; if the characteristic is $0, \mu=1)$. But $F_{G}(\sigma)$ is finite and $F_{G}(\sigma)$ contains $F_{V}(\mu)$. Thus $\operatorname{dim} V=0$ and $F_{U}(\tau)=\{1\}$. Since $U$ is the set of unipotent elements of $G[2, \S 6-06]$ and since $F_{G}(\tau)$ is algebraic, $F_{G}(\tau)$ consists of semisimple elements. 
Theorem 10. Let $F_{G}(\sigma)$ be finite and let $\sigma$ have finite order. Then $\sigma$ keeps stable a unique maximal torus $T_{\sigma}$; and $T_{\sigma}$ contains $F_{G}(\sigma)$. Moreover $T_{\sigma}$ is the only maximal torus of $G$ stable under the p-regular part $\sigma_{s}$ of $\sigma ;$ and $T_{\sigma}$ contains $F_{G}\left(\sigma_{s}\right)$.

Proof. By Proposition 8, $\sigma$ keeps stable some maximal torus $T$ of $G$. Let $g$ be in $F_{G}(\tau)$ where $\tau=\sigma_{s}$. Then since $G=T U$ where $U$ is a closed connected characteristic unipotent subgroup, $g=t u$ with $t$ in $T$ and $u$ in $U$. But $g=g^{\tau}=t^{\tau} u^{\tau}$ and $t^{\tau}$ is in $T, u^{\tau}$ in $U$. By the uniqueness of the decomposition $g=t u, t=t^{\tau}$ and $u=u^{\tau}$. Thus $u$ is in $F_{G}(\tau)$. By Lemma 9, $u=1$. Thus $g=t \in T$ and $F_{G}(\tau) \subseteq T$. Since $\tau \in\langle\sigma\rangle$, $F_{G}(\sigma) \subseteq F_{G}(\tau) \subseteq T$.

If $S$ is a second maximal torus stable under $\tau$, then $S=T^{x}$ for some $x$ in $U$. We have $T^{x}=\tau\left(T^{x}\right)=T^{\tau(x)}$ and $\tau(x) x^{-1}$ is in $N=\left\{u \in U \mid T^{u}=T\right\}$ (here we use the notation $T^{a}=a^{-1} T a$ ). Since $G$ is solvable, $N$ is a closed connected group centralizing $T$ see $[2, \S 6-04]$. Since $T$ and $U$ are $\tau$-stable and $N$ is the centralizer in $U$ of $T, N$ is $\tau$-stable. Thus $N=1_{\tau} N$ since $F_{U}(\tau)$ is finite by Lemma 9. Thus $\tau(x) x^{-1}=\tau(y) y^{-1}$ for some $y$ in $N$. Now $y^{-1} x$ is in $F_{G}(\tau)$. Since $F_{G}(\tau) \subseteq T$, we have $T^{x}=T^{y^{-1} x}=T$. Thus $T$ is the only $\tau$-stable maximal torus of $G$. It follows that $T$ is the only $\sigma$-stable maximal torus of $G$ (this last fact is proved more directly by replacing $\tau$ by $\sigma$ and $N$ by the normalizer of $T$ in $G$ in the above argument).

Theorem 11. Suppose that $G$ is not nilpotent and let $\sigma$ be of finite squarefree order. Then there exist $\sigma_{i}$ in $\langle\sigma\rangle(i=1,2)$ such that $\sigma=\sigma_{1} \sigma_{2}$, $F_{G}\left(\sigma_{1}\right)$ contains a torus of positive dimension, and $F_{G}\left(\sigma_{2}\right)$ contains a closed connected unipotent subgroup of positive dimension.

Proof. This is trivial if $F_{G}(\sigma)$ is infinite (since an infinite linear algebraic group contains either a torus of positive dimension or a closed connected unipotent subgroup of positive dimension, and a connected nonnilpotent algebraic group contains both) [2, Exposé 6].

Now let $F_{G}(\sigma)$ be finite. Then $G$ is solvable and $G=T U$ where $T$ is a $\sigma$-stable torus and $U$ is a closed connected characteristic unipotent subgroup of $G$ (Theorems 7 and 10). Define $U_{1}=\{u \in U \mid u$ is central in $U$ and $\left.u^{p}=1\right\}, \cdots, U_{i+1}=\left\{u \in U \mid u U_{i}\right.$ is central in $U / U_{i}$ and $\left.u^{p} \in U_{i}\right\}$ (at characteristic 0 this is just the ascending central series). For some $n, U=U_{n}$ (since $U$ is nilpotent by [5], and of finite exponent $p^{e}$ for some $e$ if the characteristic is $\left.p>0\right) .\langle\sigma\rangle T$ (semidirect) acts in $W_{i}=U_{i+1} / U_{i}$ for all $i \geqq 1$. Since $G$ is not nilpotent, we can choose $i$ such that $T$ acts nontrivially in $W_{i}$ (for otherwise $1 \leqq U_{1} \leqq \cdots \leqq U_{n} \leqq U_{n} T=G$ would be a central series for $G)$. Choose such an $i$ and let $W=W_{i}$. Let $T_{k}=\left\{t \in T \mid t^{k}=1\right\}$ ( $k$ a 
positive integer). Since $\bigcup_{(k,|\sigma| p)=1} T_{k}$ is dense in $T$, we can choose $k$ such that $(k,|\sigma| p)=1$ and $T_{k}$ acts nontrivially in $W$. Let $\$=\langle\sigma\rangle T_{k}$. $W$ is a vector space (over the field of $p$ elements if the characteristic is $p>0$; otherwise over the underlying field of $G$ ) and (S) acts in $W$ as a group of linear transformations. Since $B$ is finite, $W$ has a finite dimensional subspace $V$, stable under (F), on which $T_{k}$ acts nontrivially.

We now use a refinement of a technique used by Higman in [4]. Let $\bar{V}$ be the vector space obtained from $V$ by extending the ground field of $V$ to its algebraic closure. If $\alpha$ is a function from $T_{k}$ in to the underlying field of $\bar{V}$, let $\bar{V}_{\alpha}=\left\{v \in \bar{V} \mid v t=\alpha(t) v\right.$ for all $t$ in $\left.T_{k}\right\}$. Then $\bar{V}=\Sigma \oplus \bar{V}_{\alpha}$. Let $R=\left\{\alpha \mid \bar{V}_{\alpha} \neq\{0\}\right\}$. Then (S) acts in $R$ in a natural way and $R$ contains an element $\alpha$ such that $\alpha(t) \neq 1$ for some $t$ in $T_{k}$. Choose such an $\alpha$. Let $\left\langle\sigma_{1}\right\rangle$ be the isotropy subgroup of $\langle\sigma\rangle$ acting in $R$ at the point $\alpha$. Since $|\sigma|$ is squarefree, $\langle\sigma\rangle=\left\langle\sigma_{1}\right\rangle \times\left\langle\sigma_{2}\right\rangle$ (direct) for some $\sigma_{2}$ in $\langle\sigma\rangle$. $\sigma_{1}$ keeps $\bar{V}_{\alpha}$ stable whereas $\left\langle\sigma_{2}\right\rangle$ acts simply transitively on the orbit of $\alpha$ under $\langle\sigma\rangle$; and $\left(\sigma_{2}\right)^{i}$ maps $\bar{V}_{\alpha}$ onto $\bar{V}_{\alpha_{i}}$ where $\alpha_{i}$ is the image of $\alpha$ under $\left(\sigma_{2}\right)^{i}$. Let $a=\left|\sigma_{1}\right|, b=\left|\sigma_{2}\right|$. Choose $t$ in $T_{k}$ such that $\alpha(t) \neq 1$ and let $\bar{t}=\sigma_{1}(t)\left(\sigma_{1}\right)^{2}(t) \cdots\left(\sigma_{1}\right)^{a}(t)$. Choose a nonzero element $x$ in $\bar{V}_{\alpha}$ and let $\bar{x}=\sigma_{2}(x)+\left(\sigma_{2}\right)^{2}(x)+\cdots+\left(\sigma_{2}\right)^{b}(x)$. Then $\sigma_{1}(\bar{t})=\bar{t}$ and $\sigma_{2}(\bar{x})=\bar{x}$. Now $\bar{t} \neq 1$ since $t \mid \bar{V}_{\alpha}$ and $\sigma_{1} \mid \bar{V}_{\alpha}$ are commuting transformations of $\bar{V}_{\alpha}$, so that

$$
\begin{aligned}
\bar{t} \mid \bar{V}_{\alpha} & =\left(\left(\sigma_{1}\right)^{-1} t \sigma_{1}\right)\left(\left(\sigma_{1}\right)^{-2} t\left(\sigma_{1}\right)^{2}\right) \cdots\left(\left(\sigma_{1}\right)^{-a} t\left(\sigma_{1}\right)^{a}\right) \mid \bar{V}_{\alpha} \\
& =t^{a} \mid \bar{V}_{\alpha}=(\alpha(t))^{a} \operatorname{id}_{\bar{V} \alpha} \neq \operatorname{id} \bar{V} \alpha
\end{aligned}
$$

(since $\left(a,\left|T_{k}\right|\right)=1$ implies that $\left.(\alpha(t))^{a} \neq 1\right)$. And $\bar{x} \neq 0$ since the elements $\sigma_{2}(x), \cdots,\left(\sigma_{2}\right)^{b}(x)$ are linearly independent $\left(\Sigma \bar{V}_{\alpha_{i}}\right.$ is direct and $\left(\sigma_{2}\right)^{i}(x)$ lies in $\bar{V}_{\alpha_{i}}$ for all $\left.i\right)$.

Thus $F_{T_{k}}\left(\sigma_{1}\right) \neq\{1\}$ and $F_{U_{i+1} / U_{i}}\left(\sigma_{2}\right) \neq\{1\}$. It follows that $F_{G}\left(\sigma_{1}\right)$ contains a torus of positive dimension (for otherwise $F_{T}\left(\sigma_{1}\right)$ would be finite, and by Lemma $6, x^{a}=1$ for $x$ in $F_{T}\left(\sigma_{1}\right)$; but this is impossible since $\left(a,\left|T_{k}\right|\right)=1$ and $\left.F_{T}\left(\sigma_{1}\right) \geqq F_{T_{k}}\left(\sigma_{1}\right)>\{1\}\right)$. We next show that $F_{G}\left(\sigma_{2}\right)$ contains a closed connected unipotent subgroup of positive dimension. For this, let $\sigma_{2}=\tau \mu$ where $\tau, \mu$ are the $p$-regular and $p$ singular components of $\sigma_{2}$ respectively. Then $F_{U_{i+1} / U_{i}}(\tau) \neq\{1\}$. Since $H^{1}\left(\langle\tau\rangle, U_{i}\right)=\{0\}$ (the exponents of $\langle\tau\rangle$ and $U_{i}$ are relatively prime), it follows that $F_{U}(\tau) \neq\{1\}$. Thus $F_{U}(\tau)$ is infinite by Lemma 6 . Since $F_{U}(\tau)$ is $\mu$-stable, an application of Lemma 3 with $(5)=\langle\mu\rangle F_{U}(\tau)$ and $\mathfrak{S}=F_{U}(\tau)$ shows that $F_{U}\left(\sigma_{2}\right)=F_{U}(\tau \mu)=F_{\Theta}(\mu)$ is infinite. Thus $F_{G}\left(\sigma_{2}\right)$ contains a closed connected unipotent subgroup of positive dimension. 
THEOREM 12. If $\sigma$ has prime period and $F_{G}(\sigma)$ is finite, then $G$ is nilpotent.

Proof. This is immediate from Theorem 11.

We conclude with an example of some of the structure described above (which shows in particular that in Theorem 12 "prime period" cannot be replaced by "prime power period"). Let $V$ be a vector space over $F$ with basis $e_{1}, \cdots, e_{m}, f$. Let $g(a, b)$ be the linear transformation of $V$ defined by $g(a, b) e_{i}=a_{i} e_{i}+b_{i} f, g(a, b) f=f$ for $a=\left(a_{1}, \cdots, a_{m}\right), b=\left(b_{1}, \cdots, b_{m}\right)$ in $F^{m}$. Let $G=\left\{g(a, b) \mid \pi a_{i}=1\right\}$. $G$ is a group since $g(1,0)=I$ and $g(a, b) g(c, d)=g(a c, b c+d)$ (where $0=(0, \cdots, 0), 1=(1, \cdots, 1)$ and $x y, x+y$ are defined componentwise for $x, y$ in $\left.F^{m}\right)$. Let $T=\left\{g(a, 0) \mid \pi a_{i}=1\right\}, U=\left\{g(1, b) \mid b \in F^{m}\right\}$. $G=T U$ (semidirect); and $G$ is an algebraic group with maximal torus $T$ and nilradical $U$. If $m \geqq 2, G$ is not nilpotent (since $T$ is not central, see $[2, \S 6-04])$. The basis change $\left(e_{1}, \cdots, e_{m}, f\right) \rightarrow\left(\epsilon e_{m}, e_{1}, \cdots, e_{m-1}, f\right)$ $\left(\epsilon\right.$ in $F$ ) induces a birational automorphism $\sigma_{\epsilon}$ of $G$ sending $g(a, b)$ into $g\left(a^{\prime}, b^{c}\right)$ where $\left(a_{1}, \cdots, a_{m}\right)^{\prime}=\left(a_{m}, a_{1}, \cdots, a_{m-1}\right)$ and $\left(b_{1}, \cdots, b_{m}\right) \epsilon=\left(\epsilon b_{m}, b_{1}, \cdots, b_{m-1}\right)$. If $\epsilon \neq 1, F_{G}\left(\sigma_{\epsilon}\right)=\{g(a, 0) \mid a$ $=(\delta, \cdots, \delta)$ and $\left.\delta^{m}=1\right\}$ is a finite subset of $T$. If $\epsilon$ is a primitive $q$ th root of $1, \sigma$, has finite order $m q$. By varying $m$ and $q$, one finds various directions in which Theorems 11 and 12 cannot be generalized.

\section{BIBLIOGRAPHY}

1. C. Chevalley, Fondements de la gêométrie algêbrique, Faculté des Sciences de Paris, 1958.

2. - (Séminaire $\mathrm{C}$. Chevalley), Classification des groupes des Lie algêbrique, Vols. I, II, Ecole Norm. Sup. Paris, 1958.

3. D. Hertzig, The structure of Frobenius algebraic groups, Amer. J. Math. 3 (1961), 421-431.

4. G. Higman, On groups and rings which possess automorphisms without nontrivial fixed elements, J. London Math. Soc. 32 (1957), 321-324.

5. E. Kolchin, On certain concepts in the theory of algebraic matric groups, Ann. of Math. 49 (1948), 775-776.

6. D. J. Winter, On automorphisms of algebraic groups. Bull. Amer Math Soc. 72 (1966), 706-708.

YALE UNiversity 